\title{
Cesarean section under combined spinal epidural in parturient with frontal glioma
}

\author{
Caitlin VanDeCappelle, HK Ma, E Ho
}

\begin{abstract}
Our patient presented to hospital in acute asthma exacerbation and foetal distress. Past history was remarkable for pregnancy at 35 weeks, morbid obesity, uncontrolled asthma, seizure disorder and known frontal glioma. Here we highlight the neuraxial management of this patient for cesarean section and discuss the central dogma of neuraxial technique in the face of known intracranial neoplasm.
\end{abstract}

Key words: Frontal glioma, intracranial pathology and neuraxial techniques, obstetric anaesthesia

\section{INTRODUCTION}

Intracranial neoplasm has been suggested to have an incidence of 21.42 cases per 100,000 in the general population, with unchanged frequency in parturients. ${ }^{[1]}$ Glioma is the most common of intracranial neoplasms, making up about $30 \%$ of all central nervous system tumours and $80 \%$ of malignant brain tumours. ${ }^{[2]}$

Cesarean section under neuraxial anaesthesia remains the mainstay of management in North America, for concerns of increased difficult airway and aspiration risk that is inherent to parturients. Classically, however, intracranial pathology remains a contraindication to neuraxial technique due to the increased risk of herniation or worsening neurological condition in the context of raised intracranial pressure (ICP) and absence of adequate cerebrospinal fluid (CSF) flow or volume. A CSF leak could also cause a change in transmural pressure, which is

Department of Anesthesia, McMaster University, Hamilton, ON, Canada

Address for correspondence:

Dr. Caitlin VanDeCappelle,

90 Charlton Ave. W Unit 703, Hamilton, ON L8P0B4, Canada.

E-mail: caitlinvandecappelle@gmail.com

\begin{tabular}{|l|l|}
\hline \multicolumn{2}{|c|}{ Access this article online } \\
\hline Quick Response Code: & Website: \\
\hline & www.jnaccjournal.org \\
\cline { 2 - 2 } & \\
\hline
\end{tabular}

particularly worrisome in the context of a vascular tumour. Here, we present a case highlighting the conscientious use of neuraxial anaesthesia in a parturient with intracranial mass. Consent for publication was provided by the patient.

\section{CASE REPORT}

Our patient was a G3P2, 32-year-old woman admitted at $35+0$ weeks for decreased Foetal movement, non-reassuring heart rate, and asthma exacerbation. Past medical history was remarkable for asthma requiring home nebulizers, obesity with a body mass index of 53, known frontal glioma, seizure disorder, and migraines. She had been previously admitted for asthma exacerbation the month prior on two other occasions, one which was complicated by pneumonia and sepsis requiring Intensive Care Unit admission. At that time, the patient endorsed cramping and decreased foetal movements, which was difficult assess by ultrasound due to the severity of her exacerbation and size. Management for this admission consisted of nebulized salbutamol, ipratropium bromide, budesonide/formoterol, and intravenous piperacillin/tazobactam. Intubation was not required, and she was transferred to the obstetrical step-down unit

This is an open access article distributed under the terms of the Creative Commons Attribution-NonCommercial-ShareAlike 3.0 License, which allows others to remix, tweak, and build upon the work non-commercially, as long as the author is credited and the new creations are licensed under the identical terms.

For reprints contact: reprints@medknow.com

How to cite this article: VanDeCappelle C, Ma HK, Ho E. Cesarean section under combined spinal epidural in parturient with frontal glioma. J Neuroanaesthesiol Crit Care 2016;3:129-32. 
(SDU) for monitoring, after which her respiratory status stabilised. A full obstetrical assessment indicated normal foetal heart rate, positioning, and intact membranes.

The patient was previously diagnosed with low-grade frontal glioma by biopsy via burr-hole in 2010 following a year-long history of headache, nausea, vomiting, and dizzy spells. Given the context of her low-grade glioma, she did not receive further surgical interventions or radiation therapy, and she received serial imaging to assess the size of the mass.

Most recent magnetic resonance imaging of the lesion had been performed in 2013, which showed a stable, $2.6 \mathrm{~cm} \times 1.2 \mathrm{~cm} \times 1.1 \mathrm{~cm}$, increased T2-signal and ill-defined gyral expansion involving the anterior, parasagittal right frontal lobe [Figure 1]. No findings of increased mass effect or new signal abnormalities were found and midline structures remained centrally located. In anticipation of impending delivery, a computed tomography of the head was ordered by the obstetrical staff at 26 weeks gestation which highlighted a normal ventricular system, lack of midline shift, and no increase in growth. All imaging revealed a mass stable since diagnosis in 2010.

On history, our patient revealed that she received a labour epidural in 2009, just months prior to her glioma diagnosis. She became hypotensive following local anesthestic administration through the catheter, which was suspected to have traversed the dura inadvertently. According to the patient, this resulted in a motor block of the legs, but no shortness of breath or need for airway intervention. While the patient suffered from a postdural puncture headache in her post-partum period, she did not report any long-lasting neurologic sequelae following the dural puncture. Several months later, the diagnosis with low-grade glioma was made through imaging following a new-onset seizure and confirmed by biopsy.

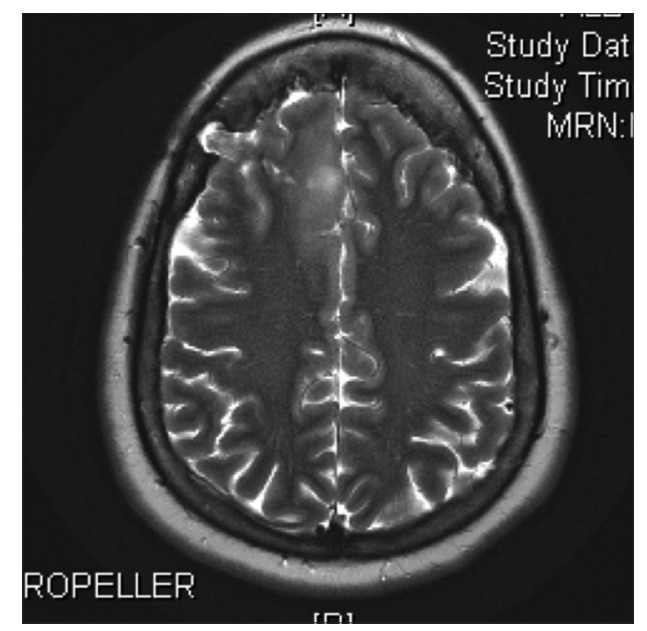

Figure 1: MRI Head showing an ill-defined gyral mass, $2.6 \times 2.1 \times 1.1 \mathrm{~cm}$ involving the anterior, parasagittal right frontal lobe
On preanaesthetic consultation, the patient was neurologically intact with a Glasgow Coma Scale of 15 . She was $168 \mathrm{~cm}$ tall and $148 \mathrm{~kg}$. Her pulse rate was 74, and blood pressure was 133/74. Airway examination revealed a thick neck, Class III Mallampati score with normal thyromental distance and mouth opening. Chest auscultation revealed normal yet decreased breath sounds, improved from the rhonchi appreciated at consultation. Blood work showed a haemoglobin of 93, platelet count of 242, international normalised ratio of 1.0, and partial thromboplastin time of 31 . Urine was negative for proteinuria. We discussed with her both general and neuraxial anaesthetic options, including awake intubation and consent was obtained. Neurosurgery was consulted, who felt it would be safe to proceed with neuraxial technique given the stability in the patient's lesion, absence of raised ICP and absence of tumour vascularity.

Due to the atypical foetal heart rate pattern and lack of accelerations, the patient went for emergent cesarean section 2 days following admission at $35+2$ days. She had remained nil per oral (NPO) for more than $8 \mathrm{~h}$ preoperatively and received $1 \mathrm{~g}$ cefazolin, $50 \mathrm{mg}$ ranitidine and sodium citrate as pre-medication. Two anaesthesiologists were present for the procedure, and based on the previous discussion with neurosurgery, avoiding difficult airway and manipulation in a patient with recent asthma exacerbation, proceeded with neuraxial technique.

A preprocedure arterial line was placed in the right radial artery. Under aseptic technique and in the sitting position, a combined spinal-epidural block was performed at the L3-L4 level using an 18-gauge Touhy and 26-gauge pencan pencil point needle. Two attempts were required to establish loss of resistance at $9.5 \mathrm{~cm}$, after which the dura was punctured, and $1.5 \mathrm{ml}$ of $0.75 \%$ Bupivacaine with $15 \mu \mathrm{g}$ fentanyl and $100 \mu \mathrm{g}$ of Epimorph were injected. The epidural catheter was then threaded easily with no complications.

A $2.38 \mathrm{~kg}$ female infant was delivered with Apgar Score of 9 at $1 \mathrm{~min}$ and 10 at $5 \mathrm{~min}$.

Blood pressure remained stable throughout the procedure. The patient required $15 \mathrm{ml}$ of $2 \%$ lidocaine with epinephrine in $5 \mathrm{ml}$ aliquots and $5 \mathrm{ml}$ of $0.125 \%$ bupivacaine with epinephrine throughout the duration of the procedure. The incision was displayed widely, necessitating several figures of eights to achieve haemostasis. As a result of this and the need for multiple layers to close subcutaneous fat, the total procedure lasted $120 \mathrm{~min}$. Two units of packed red blood cells were given to the patient to compensate for a 1500-2000 $\mathrm{ml}$ blood loss. The patient was transferred back to the SDU where her care was transferred to the obstetrical medicine staff. 
Our patient remained in the SDU for 2 days following the procedure with her epidural in situ for post-operative pain control. She and her daughter were discharged home on postoperative day 2 and 9, respectively, with regular follow-up in regards to her post-partum and neurologic care.

\section{DISCUSSION}

Neuraxial technique in parturients with intracranial pathology affords the risk of CSF leak causing herniation and clinical deterioration. While there have been many previous reports of successful neuraxial techniques for labour analgesia and cesarean section in patients with intracranial neoplasms, increases in CSF pressure cannot be confidently avoided. ${ }^{[3-9]}$ As described by Leffert and Schwamm, cases of stable, slow growing brain tumours located away from CSF pathways may cause little to no ventricular compromise due to compensatory caudal displacement of CSF or cerebral blood volume instead of brain mass. ${ }^{[10]}$ This, however, is contrasted by the suggestion of raised CSF pressure following epidural with as little as $10 \mathrm{ml}$ of bupivacaine injected into the epidural space. ${ }^{[11]}$ We, therefore, were caught in the dilemma of attempting such technique in the face of high-risk general anaesthetic.

An anaesthetic plan was derived in consultation with the neurosurgical and obstetrical teams. Consensus that frontal glioma had been stable over time and was not exhibiting current signs or symptoms of increased ICP, was made. As the tumour was not vascular in nature, close to the ventricles and had remained stable since diagnosis, the neurosurgical team felt it was reasonable to attempt a dural puncture. It was felt that such a mass would not be affected by any small to moderate change of transmural gradient as in the case of CSF leak.

In hopes of avoiding theoretical dural compression from large epidural volumes, we opted for a technique that would allow for titratable analgesia achievable with small volumes. A combined spinal-epidural technique was chosen and discussed with the patient. Risks of neuraxial technique were listed, including infection, bleeding, post-puncture headache and the possibility of herniation and worsening sequelae.

Due to our patient's morbid obesity, there was an increased chance of dural puncture with the large-bore epidural needle. It is interesting to note, however, that the incidence of dural puncture in the literature is only $1.7 \%$ for CSE as compared to $4.2 \%$ with epidural technique alone. ${ }^{[12]}$ In experienced hands, this risk has been suggested to be as low as $0.2 \% \cdot{ }^{[12]}$ Given this risk, we chose to puncture the dura with much smaller pencil-point needle in order to minimise CSF leak.
Anaesthesia for parturients with brain tumours or potential for raised ICP must be approached with caution due to the increased risk of brain herniation, tumor haemorrhage or seizure. ${ }^{[7]}$ Both general and neuraxial anesthetic options continue to be controversial. In addition to risk of aspiration and difficult airway in parturients, general anaesthesia can also be associated with an acute rise in ICP during tracheal intubation and extubation. Neuraxial technique, either via spinal anaesthesia or an inadvertent dural puncture during epidural anaesthesia, can cause CSF leak, leading to brain herniation and/or neurologic sequelae though avoids the physiologic stress of intubation. In our case, the patient's physical examination, imaging, medical records, and multidisciplinary discussion reassured us that there was no obvious rise in ICP, and her tumor was stable. By opting for a neuraxial technique, we avoided the risk of bronchospasm following awake fibreoptic intubation in a patient with recent asthma exacerbation.

Here, we have presented a case to challenge the previous dogma of avoiding neuraxial technique for patients with intracranial masses in a morbidly obese, asthmatic patient with frontal lobe tumor requiring urgent cesarean section. We suggest that the decision between general or regional anaesthesia should be made on an individual patient basis in collaboration with the patient, neurosurgical, and obstetrical teams.

\section{Financial support and sponsorship}

Nil.

\section{Conflicts of interest}

There are no conflicts of interest.

\section{REFERENCES}

1. CBTRUS Fact Sheet. Central Brain Tumour Registry of the United States (CBTRUS). Available from: http://www.cbtrus. org/factsheet/factsheet.html. [Last accessed on 2015 Aug 14].

2. Goodenberger ML, Jenkins RB. Genetics of adult glioma. Cancer Genet 2012;205:613-21.

3. Atanassoff PG, Alon E, Weiss BM, Lauper U. Spinal anaesthesia for caesarean section in a patient with brain neoplasma. Can J Anaesth 1994;41:163-4.

4. Chhabra A, Kumar N, Kumar A, Singh N, Sharma BS. Combined spinal epidural anesthesia for cesarean section in a pregnant patient with rare intracranial neoplasm. Middle East J Anaesthesiol 2010;20:581-4.

5. Norris MC, Grieco WM, Borkowski M, Leighton BL, Arkoosh VA, Huffnagle HJ, et al. Complications of labor analgesia: Epidural versus combined spinal epidural techniques. Anesth Analg 1994;79:529-37.

6. Shigeki S, Ryoichi O, Shin'ichi N, Chiemi N, Tatsuya Y, Junzo T. Anesthetic management for elective cesarean section in patient with a coexisting brain tumor. J Clin Anesth 2003;27:1645-6.

7. Hirs I, Grbcic P. Cesarean section in spinal anesthesia on a patient with mesencephalic tumor and ventriculoperitoneal 
drainage - A case report. Korean J Anesthesiol 2012;63:263-5.

8. Shah PN, Sonawane D, Appukutty J. Anaesthetic management for caesarean section in a case of previously operated with residual pituitary tumour. Indian J Anaesth 2011;55:618-20.

9. Chang L, Looi-Lyons L, Bartosik L, Tindal S. Anesthesia for cesarean section in two patients with brain tumours. Can J Anaesth 1999;46:61-5.
10. Leffert LR, Schwamm LH. Neuraxial anesthesia in parturients with intracranial pathology: A comprehensive review and reassessment of risk. Anesthesiology 2013;119:703-18.

11. Semple DA, McClure JH. Arnold-chiari malformation in pregnancy. Anaesthesia 1996;51:580-2.

12. Finfer SR. Management of labour and delivery in patients with intracranial neoplasms. Br J Anaesth 1991;67:784-7. 